Pacific Journal of Mathematics

NEW PROOFS OF BING'S APPROXIMATION THEOREMS FOR 


\title{
NEW PROOFS OF BING'S APPROXIMATION THEOREMS FOR SURFACES
}

\author{
J. W. CANNON
}

We give alternative proofs of Bing's Side Approximation and Homeomorphic Approximation Theorems for surfaces in 3-manifolds.

o. Introduction. We give in $\$ 1$ an alternative proof of Bing's Side Approximation Theorem (S.A.T.) [4] [7] [8]:

S.A.T. Suppose that $S$ is a 2-sphere topologically embedded in $E^{3}$. Then, for each $\varepsilon>0$, there are disjoint $\varepsilon$-disks $D_{1}, \cdots, D_{n}$ in $S$ and an $\varepsilon$-homeomorphism $h$ from $S$ into $E^{3}$ such that

(1) $h(S)$ is polyhedral, and

(2) $S-\bigcup_{i} D_{i} \subset \operatorname{Ext} h(S)$.

One may require alternatively that

( $\left.2^{\prime}\right) \quad S-\bigcup_{i} D_{i} \subset \operatorname{Int} h(S)$.

In $\S 2$ we deduce from the above the slightly stronger version of the S.A.T. (also due to Bing) which one generally encounters in applications.

In the strict sense, Bing's proof of the S.A.T. is completely elementary. Our proof on the other hand uses freely local homology and homotopy properties, Dehn's lemma and related theorems, and homology linking theory. We have attempted to substitute simplicity of outline for simplicity of technique. This has the advantage that the outline serves almost without change in a proof of the Moise [15]-Bing [6] theorem on the triangulability of 3-manifolds and the Armentrout [3] theorem on approximating cellular maps between 3-manifolds by homeomorphisms. We do not go into detail on these latter theorems. Some details of the proof which are unique to the S.A.T. (and which, in fact, make it harder than the triangulation and cellular map theorems) have been proved in their more natural setting, "ULC properties of embedded curves and surfaces in $E^{33 "}$ [10, $\S 2$ and the first half of $\S 3]$. This allows us to make the outline even more transparent and unencumbered by detail. The necessary information from [10] is summarized in four lemmas at the end of this introduction. This quick summary allows the present paper to be read independently of [10]. We give a short proof in $\$ 3$ of another of Bing's important theorems on surfaces, namely, the Homeomorphic Approximation Theorem (H.A.T.) [5]. The shortness arises from the fact that we use the S.A.T. to do the work that Bing did from scratch.

H.A.T. Suppose that $S$ is a topologically embedded 2-sphere in 
$E^{3}$ and that, for each $\varepsilon>0$, there is an $\varepsilon$-homeomorphism from $S$ into Int $S$. Then $S \cup \operatorname{Int} S$ is a 3-cell.

Papers [9] and [10] are the basic references for this paper: Reference [9] includes descriptions of the Euclidean spaces $\left(E^{1}, E^{2}, E^{3}, \cdots\right)$, $n$-cells $\left(B^{0}, B^{1}, B^{2}, \cdots\right.$ and their homeomorphic images), $n$-spheres ( $S^{0}=\mathrm{Bd} B^{1}, S^{1}=\mathrm{Bd} B^{2}, \cdots$ and their homeomorphic images), complexes, manifolds, disks (2-cells), arcs (1-cells), simple closed curves and loops, finite graphs (1-complexes), polyhedra, and general position and cut-and-paste techniques. It also gives statements of Dehn's Lemma [16] (cf. [9, 4.5.1 and Addendum to 4.5.1], the Loop Theorem [17], and the Sphere Theorem [16]). Such concepts are widely known and need only be checked by the beginner. Reference [10] contains technical material less widely known and summarized here:

Notation. We use $\rho$ for the Euclidean metric, Diam for diameter, $\mathrm{Cl}$ for closure, Bd for boundary (point set or combinatorial), Int and Ext for interior and exterior, $N(X, \varepsilon)$ for the (open) $\varepsilon$-neighborhood of $X$ in $E^{3}$. An $\varepsilon$-set has diameter less than $\varepsilon$; an $\varepsilon$-map or homeomorphism moves no point as far as $\varepsilon$. If $T$ is a triangulation (possibly curvilinear) of some topologically embedded polyhedron, then $T^{i}$ denotes the $i$-skeleton of $T$, and $\left|T^{i}\right|$ denotes the underlying point set which carries $T^{i}$ (i.e., the union of the simplexes in $T^{i}$ ).

Property $U L C^{n}$. Let $A$ be a subset of $E^{3}$ and $n$ a nonnegative integer. Then $A$ is $U L C^{n}$ if the following condition is satisfied: For each $\varepsilon>0$, there is a $\delta>0$ such that if $0 \leqq i \leqq n$ and $f: S^{i} \rightarrow A$ is a map from the $i$-sphere $S^{i}$ into a $\delta$-subset of $A$, then there is a map $f^{*}: B^{i+1} \rightarrow A$ into an $\varepsilon$-subset of $A$ which extends $f$ (recall that $\left.S^{i}=\mathrm{Bd} B^{i+1}\right)$.

0.1. [19, p. 66]. If $S$ is a 2-sphere in $E^{3}$ and $U$ is a component of $E^{3}-S$, then $U$ is $U L C^{0}$.

0.2. Existence of nice arcs in 2-spheres. Suppose $S$ is a 2-sphere in $E^{3}, U_{1}$ and $U_{2}$ are the components of $E^{3}-S, G$ is a finite graph in $S$, and $\varepsilon>0$. Then there is an $\varepsilon$-homeomorphism $h: S \rightarrow S$ such that $\mathrm{Cl}\left(U_{1}\right)-h(G)$ and $\mathrm{Cl}\left(U_{2}\right)-h(G)$ are $U L C^{2}$.

Proof. [10; 2C. 7(2). 2, 2C. 7(3). 2, 2C. 7(1), 2C. 3].

0.3. Suppose $S$ is a 2-sphere in $E^{3}, U_{1}$ and $U_{2}$ are the components of $E^{3}-S$, and $J$ is a simple closed curve in $S$ such that $\mathrm{Cl}\left(U_{1}\right)-J$ and $\mathrm{Cl}\left(U_{2}\right)-J$ are $U L C^{2}$. Then $J$ pierces an almost-polyhedral disk at each point of $J$. (A 2-manifold is said to be almost-polyhedral if it is locally polyhedral except at finitely many points.) 


\section{Proof. $[10 ; 3.2,3.3]$.}

0.4. Adjustments of maps in ULC sets. Suppose $C \subset E^{3}$ and $C$ is $U L C^{n}$ ( $n$ a nonnegative integer). Suppose $P$ is an $(n+1)$-complex, $B$ is a closed subset of $P$, and $F: P \rightarrow \mathrm{Cl} C$ is a map. Then, for each $\varepsilon>0$, there is a map $f^{*}: P \rightarrow \mathrm{Cl} C$ such that

$$
\begin{gathered}
f^{*}|B=f| B, \\
f^{*}(P-B) \subset C, \text { and } \\
\rho\left(f^{*}(x), f(x)\right)<\varepsilon, \text { for each } x \in P .
\end{gathered}
$$

Proof. $\quad[10,2$ C. 2].

The following information on linking is classical and is included for the use of the reader in verifying certain intuitive claims made in the proof of the S.A.T. and H.A.T. We give one example of such a verification in an appendix following $\S 3$.

Linking of Simple Closed Curves in $E^{3}$ (cf. [1, Chapter 15] and $[18, \S 77]$ for details). We use the notation $L(J, K)$ for the (homological) linking number (integer coefficients) of disjoint oriented simple closed curves or loops in $E^{3}$. The integer $L(J, K)$ may be calculated as follows. Choose polygonal oriented simple closed curves $J^{\prime}$ and $K^{\prime}$ such that $J$ is homologous to $J^{\prime}$ in $E^{3}-K$ and $K$ is homologous to $K^{\prime}$ in $E^{3}-J^{\prime}$. Let $D$ be a polyhedral singular oriented surface in $E^{3}$ bounded by the oriented curve $J^{\prime}$ and in general position with respect to $K^{\prime}$. Assume $E^{3}$ oriented. Then each oriented simplex in $D$ can be assigned a positive and a negative side in $E^{3}$ (intuitively, by the right-hand rule). Then $L(J, K)$ is the algebraic number of times $K^{\prime}$ cuts through $D$ : an intersection at which the oriented $K^{\prime}$ passes from the negative to positive side of $D$ is counted as +1 , from the positive to the negative as -1 . Then the following properties hold.

0.5. $L(J, K)=L(K, J)$ and this integer is independent of the choice of $J^{\prime}, K^{\prime}$, and $D$ (in particular depends only on the homology class $[K]$ of $K$ in $\left.E^{3}-J\right)$.

0.6. If $J$ is an oriented simple closed curve in $E^{3}$, then there is an isomorphism $H_{1}\left(E^{3}-J ; \boldsymbol{Z}\right) \simeq \boldsymbol{Z}$ which sends $[K]$ to $L(J, K)$.

0.7. If $D$ is a disk in $E^{3}$ (not necessarily polyhedral) and $p \in \operatorname{Int} D$, then there is a simple closed curve $J$ in $\left(E^{3}-D\right) \cup\{p\}$ for which $L(J, \mathrm{Bd} D) \neq 0$. Furthermore, if $J$ is any such loop and $J_{1}$ is any loop in $E^{3}-\operatorname{Bd} D$, then $J_{1}$ is homologous in $E^{3}-\mathrm{Bd} D$ to some multiple of $J$. In particular $|L(J, \mathrm{Bd} D)|=1$ by $(0.6)$. 
Finally, we mention that we shall use freely the standard cutand-paste procedures of 3-space topology without being too explicit about the details. For the uninitiated (and even for the initiated who finds the details of the proof of Lemma (4.2), §4, somewhat outside the range of his experience) we recommend the note by Griffith and Harrold in the Proceedings of the National Academy of Science [12] where the procedure is carried out in some detail. (Note the comments about [12] at the end of our paper.)

1. Proof of the S.A.T. Throughout this section, $S$ will denote a topologically embedded 2-sphere in Euclidean 3-dimensional space $E^{3}$ and $\varepsilon$ a positive number.

Choose a triangulation $T$ of $S$ which has mesh so small that the simplicial neighborhood in $T$ of each simplex has diameter less than $\varepsilon / 8$. By (0.2), we may assume that the carrier $G=\left|T^{1}\right|$ of the 1-skeleton $T^{1}$ of $T$ is a finite graph such that $\mathrm{Cl}($ Int $S)-G$ and $\mathrm{Cl}($ Ext $S)-G$ are $U L C^{2}$.

Step 1. Building an almost-polyhedral 2-sphere $S(v)$ about a vertex $v$ of $T$. We choose from the interior of the carrier of each 1 -simplex $\sigma$ of $T$ a point $p(\sigma)$. By (0.3), there is a very small almostpolyhedral disk $D(\sigma)$ pierced by $\left|T^{1}\right|$ at $p(\sigma)$. Let $v$ be a vertex of $T$ and $\sigma_{1}, \cdots, \sigma_{n}=\sigma_{0}$ the 1 -simplexes of $T$ which emanate from $v$. We assume $\sigma_{1}, \cdots, \sigma_{n}=\sigma_{0}$ indexed so that $\sigma_{i}$ and $\sigma_{j}$ are faces of a common 2 -simplex $\tau_{i j}$ of $T$ if $i-j=1$. Let $s_{i}$ be the arc in $\sigma_{i}$ bounded by $v$ and $p\left(\sigma_{i}\right)$. Let $q\left(\sigma_{i}\right)$ be a point of $\left(\operatorname{Int} \sigma_{i}\right)-s_{i}\left(q\left(\sigma_{n}\right)=\right.$ $\left.q\left(\sigma_{0}\right)\right)$. Let $s_{i}^{\prime}$ be the arc in $\sigma_{i}$ bounded by $v$ and $q\left(\sigma_{i}\right)$. Let $A_{i j}(i-j=1)$ denote a spanning arc of $\tau_{i j}$ with endpoints $q\left(\sigma_{i}\right)$ and $q\left(\sigma_{j}\right)$. We require that $A_{i j}$ be very near homeomorphically to the arc $s_{i}^{\prime} \cup s_{j}^{\prime}$. Then $J=\bigcup_{i-j=1} A_{i j}$ is a simple closed curve in $S$ which bounds a small disk

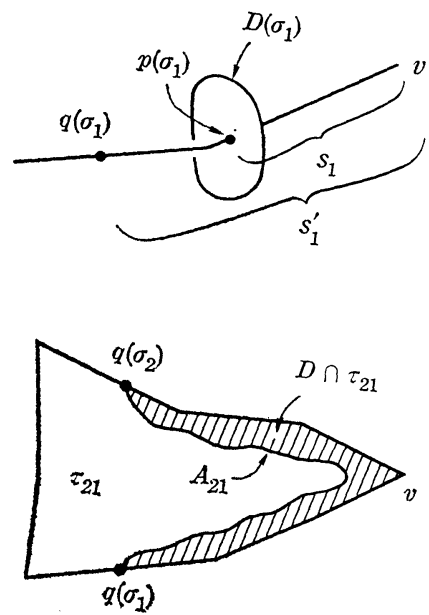

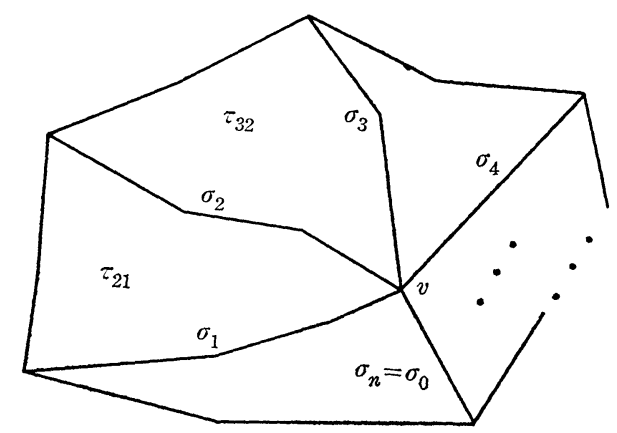

FIGURE 1 
$D$ in $S$ with $\bigcup_{i} s_{i} \subset \operatorname{Int} D$. We may require that $D \cap D\left(\sigma_{i}\right)=D \cap \operatorname{Int} D\left(\sigma_{i}\right)$ and that this intersection lie very close to $p\left(\sigma_{i}\right)$. (See Figure 1.)

Since $\mathrm{Cl}(\operatorname{Int} S)-\left|T^{1}\right|$ and $\mathrm{Cl}(\operatorname{Ext} S)-\left|T^{1}\right|$ are $U L C^{1}$, it follows from (0.4) that there are maps $f: D \rightarrow S \cup \operatorname{Int} S$ and $g: D \rightarrow S \cup$ Ext $S$ which fix $\mathrm{Bd} D$, move points of Int $D$ only very slightly, and take Int $D$ into the complement of $\left|T^{1}\right|$. We may think of $f(D) \cup g(D)$ as a singular 2-sphere in $E^{3}$. After slight adjustment we may assume that $f(D) \cup g(D)$ is in general position with respect to $\cup_{i} \mathrm{D}\left(\sigma_{i}\right)$. We require that $f$ and $g$ be chosen so near to the identity that $(f(D) \cup g(D)) \cap \bigcup_{i} \mathrm{Bd} D\left(\sigma_{i}\right)=\varnothing$. We leave it to the reader to establish the following claim (e.g., by a linking argument):

Claim. If, for each $i$ and $j$ with $i-j=1$, the arc $A_{i j}$ is very close homeomorphically to the arc $s_{i}^{\prime} \cup s_{j}^{\prime}$ and if the maps $f$ and $g$ are very near the identity map, then there is in $f(D) \cup g(D)$ (as adjusted for general position) a singular disk-with-holes $D_{0}$ which has the following properties:

(1) $D_{0} \cap \mathrm{U}_{i} \mathrm{D}\left(\sigma_{i}\right)=\mathrm{Bd} D_{0}$, and

(2) $D_{0}$ has in each $D\left(\sigma_{i}\right)$ precisely one boundary curve which is not homotopically trivial in $D\left(\sigma_{i}\right)-\left\{p\left(\sigma_{i}\right)\right\}$, and that boundary curve is homotopic in $D\left(\sigma_{i}\right)-\left\{p\left(\sigma_{i}\right)\right\}$ to a simple closed curve.

If we start with the disk-with-holes $D_{0}$ supplied by the claim, then the boundary curves of $D_{0}$ which are trivial in some $D\left(\sigma_{i}\right)$ $\left\{p\left(\sigma_{i}\right)\right\}$ may be filled in by singular disks in $D\left(\sigma_{i}\right)-\left\{p\left(\sigma_{i}\right)\right\}$, and these singular disks may then be pushed slightly to one side of $D\left(\sigma_{i}\right)$ so that (1) and (2) are still satisfied but so that the nontrivial boundary curves promised by (2) are the only boundary curves of the adjusted $D_{0}$. These nontrivial boundary curves may in turn be replaced by simple closed curves $J_{i}$ in $D\left(\sigma_{i}\right)-\left\{p\left(\sigma_{i}\right)\right\}$, by (2), so that (1) and (2) are still satisfied. We continue to call the adjusted disk-with-holes $D_{0}$.

Let $D_{i}(i=1, \cdots, n)$ be the disk in $D\left(\sigma_{i}\right)$ bounded by $J_{i}$. Then $D_{0} \cup D_{1} \cup \cdots \cup D_{n}$ is an almost-polyhedral singular 2-sphere in $E^{3}$ with no singularities near $p\left(\sigma_{1}\right), \cdots, p\left(\sigma_{n}\right)$. By Dehn's Lemma([16]; note also [9, Addendum to Theorem 4.5.1]), there is a nonsingular 2-sphere $S(v)$ in the union of $D_{1} \cup \cdots \cup D_{n}$ and an arbitrarily small neighborhood of Int $D_{0}$ such that $v \varepsilon \operatorname{Int} S(v)$ and such that $S(v)$ is polyhedral except possibly at the points $p\left(\sigma_{1}\right), \cdots, p\left(\sigma_{n}\right)$. It follows that, if $D_{0}$ is sufficiently close to $D$, we must have $S(v) \cap\left|T^{1}\right|=$ $\left\{p\left(\sigma_{1}\right), \cdots, p\left(\sigma_{n}\right)\right\}$ with $\left|T^{1}\right|$ piercing $S(v)$ at each of the points $p\left(\sigma_{i}\right)$.

Step 2. Building a polyhedral handlebody $H$ about $\left|T^{1}\right|$. It is clear from the construction described in Step 1 that, if $v_{1}, \cdots, v_{m}$ are the vertices of $T$, then we may choose the 2 -spheres $S\left(v_{1}\right), \cdots, S\left(v_{m}\right)$ so as to satisfy the following requirements: 
(3) $\left[S\left(v_{i}\right) \cup \operatorname{Int} S\left(v_{i}\right)\right] \cap\left[S\left(v_{j}\right) \cup \operatorname{Int} S\left(v_{j}\right)\right]=S\left(v_{i}\right) \cap S\left(v_{j}\right)(i \neq j)$; and this intersection is either $\varnothing$ or it is a subdisk of some $D(\sigma)$ and contains the corresponding $p(\sigma)$.

(4) $\left[S\left(v_{i}\right) \cup \operatorname{Int} S\left(v_{i}\right)\right] \cap \tau \neq \varnothing$ if and only if $v_{i}$ is a vertex of $\tau$, for each $\tau \in T$.

(5) Diam $S\left(v_{i}\right)<\varepsilon / 8$.

Let $H=\bigcup_{i=1}^{m} S\left(v_{i}\right) \cup \operatorname{Int} S\left(v_{i}\right)$. We see that $H$ is a polyhedral handlebody as follows. Suppose $S\left(v_{i}\right) \cap S\left(v_{j}\right)(i>j)$ is nonempty, hence a disk $D_{i j}^{\prime}$. Then $D_{i j}^{\prime}$ is polyhedral modulo its one-point intersection with $\left|T^{1}\right|$. Use Dehn's lemma to replace $D_{i j}^{\prime}$ by a polyhedral disk $D_{i j}$ which differs from $D_{i j}^{\prime}$ only very near $D_{i j}^{\prime} \cap\left|T^{1}\right|$. The iteration of this process changes each $S\left(v_{i}\right)$ into a polyhedral 2-sphere $T\left(v_{i}\right)$. Then $H=\bigcup_{i=1}^{m} T\left(v_{i}\right) \cup \operatorname{Int} T\left(v_{i}\right)$, and $H$ is clearly a handlebody since each of the sets $C\left(v_{i}\right)=T\left(v_{i}\right) \cup \operatorname{Int} T\left(v_{i}\right)$ is a polyhedral 3-cell. If $v_{r}, v_{s}$, and $v_{t}(r>s>t)$ are the vertices of a single 2-simplex $\tau$ in $T$, then

$$
X(\tau)=C\left(v_{r}\right) \cup C\left(v_{s}\right) \cup C\left(v_{t}\right)
$$

is a polyhedral solid torus. The disks $D_{r s}, D_{s t}$, and $D_{r t}$ are called the distinguished meridional disks of $X(\tau)$ and the other disks $D_{i j}$ which lie on $\operatorname{Bd} X(\tau)$ are called the distinguished boundary disks of $X(\tau)$. The totality of disks $D_{i j}$ are called the distinguished disks of $H$.

Step. 3. Spanning polyhedral disks across the "holes" in $H$. Let $\tau$ and $X(\tau)$ be as in the preceding paragraph. We may certainly require that $\mathrm{Bd} \tau \subset \operatorname{Int} X(\tau)$ and that $\mathrm{Bd} \tau$ be homotopic in Int $X(\tau)$ to a centerline of $X(\tau)$. As one consequence we find that there are simple closed curves $J$ and $K$ in Int $\tau$ such that $\mathrm{Bd} \tau$ and $K$ bound an annulus $A$ in $\tau \cap \operatorname{Int} X(\tau)$ with $J$ separating $\mathrm{Bd} \tau$ from $K$ in $A$. By (0.2), we may assume that $\mathrm{Cl}(\operatorname{Int} S)-K$ and $\mathrm{Cl}(\operatorname{Int} S)-K$ are $U L C^{1}$. Let $D$ be the subdisk of $\tau$ bounded by $J$. By $(0.4)$, there are maps $f: D \rightarrow S \cup \operatorname{Int} S$ and $g: D \rightarrow S \cup \operatorname{Ext} S$ which fix Bd $D$, move points of Int $D$ only slightly, and take Int $D$ into the complement of $K$. We think of $f(D) \cup g(D)$ as a singular 2-sphere and use the Sphere Theorem [16] to replace $f(D) \cup g(D)$ by a nonsingular polyhedral 2-sphere $S(\tau)$ which lies arbitrarily close to $f(D) \cup g(D)$ and has $K$ in its interior. We may assume that $S(\tau)$ is in general position with respect to $\mathrm{Bd} H$. We leave it to the reader to establish the following claim. (Again we suggest a linking argument. Cf. also the argument involving continua $K_{i}$ later in this section: if a curve in $\operatorname{Bd} D_{0}(\tau)$ bounds a disk in $\mathrm{Bd} X(\tau)$, then it does so in $(\mathrm{Bd} H) \cap X(\tau)$.)

Claim. If $f$ and $g$ are sufficiently near the identity and $S(\tau)$ is 
sufficiently near $f(D) \cup g(D)$, then there is a disk-with-holes $D_{0}(\tau)$ in $S(\tau)$ which has the following properties:

(6) $D_{0}(\tau) \cap H=\mathrm{Bd} D_{0}(\tau)=D_{0}(\tau) \cap X(\tau)$;

(7) $D_{0}(\tau)$ has precisely one boundary component which does not bound a disk in $(\mathrm{Bd} H) \cap X(\tau)$ and that component is homotopic in $X(\tau)$ to $\mathrm{Bd} \tau$.

Let $\tau_{1}, \cdots, \tau_{p}$ be the 2 -simplexes of $T$ and $D_{0}\left(\tau_{1}\right), \cdots, D_{0}\left(\tau_{p}\right)$ diskswith-holes whose existence is assured by the construction just described and the claim. We may clearly assume the following conditions satisfied:

(8) $D_{0}\left(\tau_{i}\right) \cap D_{0}\left(\tau_{j}\right)=\varnothing$ if $i \neq j$;

(9) $\operatorname{Diam}\left[D_{0}\left(\tau_{i}\right) \cup X\left(\tau_{i}\right)\right]<\varepsilon / 2$.

We now proceed to adjust the $D_{0}\left(\tau_{i}\right)$ 's so that they are disks. If the $D_{0}\left(\tau_{i}\right)$ 's are not already disks, then there is an $i$ such that one of the boundary components of $D_{0}\left(\tau_{i}\right)$ bounds a disk $E$ in $(\mathrm{Bd} H) \cap X\left(\tau_{i}\right)$ (cf.(7) above) and (Int $E) \cap \bigcup_{j} D_{0}\left(\tau_{j}\right)=\varnothing$. Add $E$ to $D_{0}\left(\tau_{i}\right)$ and push that part of the new $D_{0}\left(\tau_{i}\right)$ which is near $E$ slightly into $E^{3}-H$. An iteration of this procedure changes each $D_{0}\left(\tau_{i}\right)$ into a disk. Note that we may maintain conditions (8) and (9).

We now adjust the disks $D_{0}\left(\tau_{1}\right), \cdots, D_{0}\left(\tau_{p}\right)$ so that, for each $i$, $\mathrm{Bd} D_{0}\left(\tau_{i}\right)$ runs "straight around" $\mathrm{Bd} X\left(\tau_{i}\right)$ in $\mathrm{Bd} H$. The problem here is descriptive rather than inherent. We therefore urge the reader to examine carefully Figures 2 and 3 before attempting to read the description which follows. We fix for consideration a 2 -simplex $\tau$ of

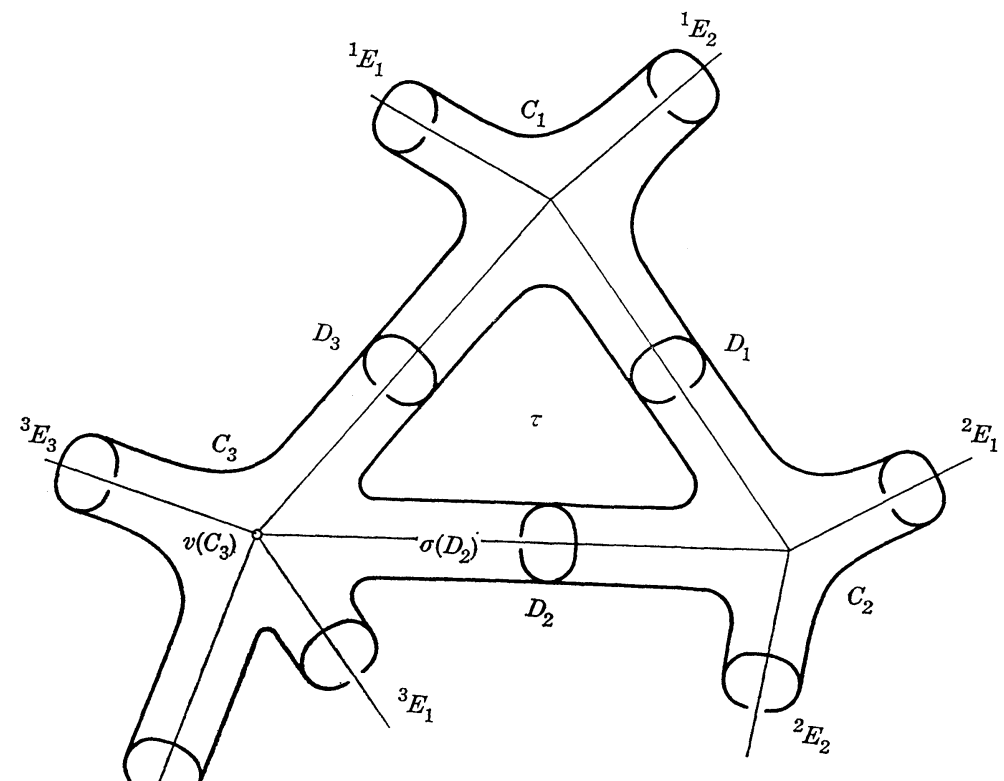

FIGURE 2 


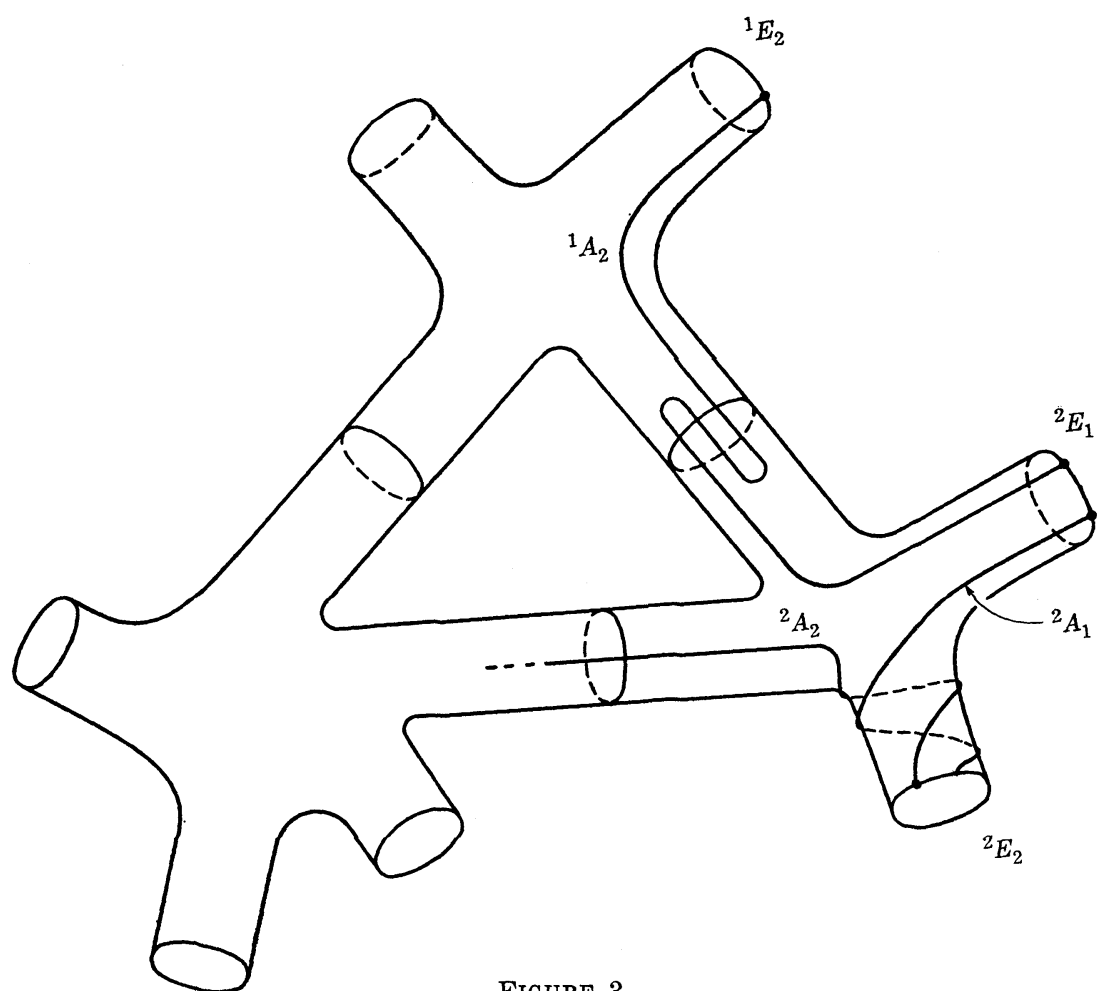

FIGURE 3

T. Recall that $X(\tau)$ is a solid torus divided by its distinguished meridional disks, which we denote by $D_{1}, D_{2}$, and $D_{3}$, into 3-cells $C_{1}, C_{2}$, and $C_{3}$, where $D_{1}=C_{1} \cap C_{2}, D_{2}=C_{2} \cap C_{3}$, and $D_{3}=C_{3} \cap C_{1}$. We denote the distinguished boundary disks of $X(\tau)$ by

$$
{ }^{1} E_{1}, \cdots,{ }^{1} E_{r(1)},{ }^{2} E_{1}, \cdots,{ }^{2} E_{r(2)},{ }^{3} E_{1}, \cdots,{ }^{3} E_{r(3)},
$$

where those with superscript $i$ lie in $\mathrm{Bd} C_{i}$.

Each of the cells $C_{i}$ encloses a unique vertex $v\left(C_{i}\right)$ of $T$; each distinguished disk $\underline{D}$ of $H$ intersects a unique 1-simplex $\sigma(\underline{D})$ of $T$. We assume the indices on the distinguished disks chosen so that each successive pair from the sequence

(10) $\sigma\left({ }^{1} E_{1}\right), \cdots, \sigma\left({ }^{1} E_{r(1)}\right), \sigma\left({ }^{2} E_{1}\right), \cdots, \sigma\left({ }^{2} E_{r(2)}\right), \sigma\left({ }^{3} E_{1}\right), \cdots, \sigma\left({ }^{3} E_{r(3)}\right)$, $\sigma\left({ }^{1} E_{1}\right)$ is a pair of edges from a single 2-simplex of $T$. It follows from (7) that there are disjoint ares ${ }^{1} A_{1}, \cdots,{ }^{1} A_{r(1)},{ }^{2} A_{1}, \cdots,{ }^{2} A_{r(2)}$, ${ }^{3} A_{1}, \cdots,{ }^{3} A_{r(3)}$ in $\mathrm{Bd} X(\tau)$ joining successive pairs of distinguished disks from the list (10) (with $\sigma$ 's removed) and lying, for each pair, in $\operatorname{Bd} D_{0}\left(\tau^{\prime}\right)$, where $\tau^{\prime}$ is the 2-simplex of $T$ which has the corresponding pair of edges in its boundary. Note that a single component $K_{i}$ of $C_{i} \cap\left(\bigcup^{j} A_{k} \bigcup^{j} E_{k}\right)$ contains the disks $\bigcup_{k}{ }^{i} E_{k}$ and intersects both of the distinguished meridional disks of $X(\tau)$ which lie in $\mathrm{Bd} C_{i}$. With our 
descriptive apparatus finally set up, we can define our task precisely: reduce $D_{0}(\tau) \cap\left(D_{1} \cup D_{2} \cup D_{3}\right)$ to exactly three points. The procedure is straightforward and is contained in the next paragraph.

We may suppose $\mathrm{Bd} D_{0}(\tau)$ in general position with respect to the union $\mathrm{Bd} D_{1} \cup \mathrm{Bd} D_{2} \cup \mathrm{Bd} D_{3}$. If the intersection of $\mathrm{Bd} D_{0}(\tau)$ with this union consists of more than three points, then since $B d D_{0}(\tau)$ is homotopic in $X(\tau)$ to a centerline of $X(\tau)$, there is an arc $A$ in $\operatorname{Bd} D_{0}(\tau)$ which lies in a single $\mathrm{C}_{i}$ and has its endpoints on a single $\mathrm{Bd} D_{j}$. The existence of the continuum $K_{i}$ (see the previous paragraph) forces $A$ to bound, together with an arc in $\mathrm{Bd} D_{j}$, a disk $E$ in $\mathrm{Bd} C_{i}$ whose interior misses each distinguished disk in $H$. Then each of the sets $D_{0}\left(\tau_{k}\right)$ which intersects $E$ can be adjusted by cut and paste near $E$ so as to have no intersection with $E$. In particular, this reduces the number of intersections of $D_{0}(\tau)$ with $\mathrm{Bd} D_{j}$ by at least two. Note that these changes may be made so as to preserve the essential properties of the disks $D_{0}\left(\tau_{1}\right), \cdots, D_{0}\left(\tau_{p}\right)$ as described in (6), (7), (8), (9), and the paragraph following (9). The reduction of $D_{0}(\tau) \cap\left(D_{1} \cup D_{2} \cup D_{3}\right)$ to three points therefore follows by induction (for each $\tau$ ).

Step 4. Building a polyhedral e-approximation to $S$. On the boundary of each distinguished disk $D_{j}$ of $H$ there are precisely two points which lie in $\mathrm{U}_{k} D_{0}\left(\tau_{k}\right)$. Let $A_{j}$ be a polygonal spanning arc of $D_{j}$ which has those two points as endpoints. Let $C_{i}$ be any of the polyhedral cells of which $H$ is composed. Then $\left[\bigcup_{j} A_{j} \cup \bigcup_{k} D_{0}\left(\tau_{k}\right)\right] \cap C_{i}$ is a single polygonal simple closed curve $J_{i}$ in $\mathrm{Bd} C_{i}$. Let $P_{i}$ be a polyhedral spanning disk of $C_{i}$ with boundary $J_{i}$. Then $P=$ $\left(\bigcup_{i} P_{i}\right) \cup\left(\bigcup_{k} D_{0}\left(\tau_{k}\right)\right)$ is a polyhedral 2-sphere that is homeomorphically within $\varepsilon$ of $S$. We leave the easy construction of the homeomorphism to the reader.

Step 5. Identifying polyhedral ع-side approximations to $S$. One sees immediately that the polyhedral 2-sphere $P$ of Step 4 locally separates $\mathrm{Bd} H$ into two components. Since $P$ is a 2-sphere in $E^{3}$ (as opposed to a one-sided surface in a 3-manifold), the separation is also global. Let $H_{1}$ and $H_{2}$ be the components of $\mathrm{Bd} H-P$ in Int $P$ and Ext $P$, respectively. Then

$$
S_{1}=H_{1} \cup\left(\bigcup_{k} D_{0}\left(\tau_{k}\right)\right) \text { and } S_{2}=H_{2} \cup\left(\bigcup_{k} D_{0}\left(\tau_{k}\right)\right)
$$

are polyhedral 2-spheres which also homeomorphically within $\varepsilon$ of $S$. Let $D_{k}$ be a subdisk of $\tau_{k}$ in Int $\tau_{k}$ which contains $\left(S_{1} \cup S_{2}\right) \cap \tau_{k}$. Then the disks $D_{1}, \cdots, D_{p}$ are the $\varepsilon$-disks whose existence is asserted by the S.A.T. and $S_{1}$ and $S_{2}$ satisfy 


$$
S-\bigcup_{k} D_{k} \subset \operatorname{Ext} S_{1}
$$

and

$$
S-\bigcup_{k} D_{k} \subset \operatorname{Int} S_{2}
$$

2. Improving the S.A.T. The following lemma is an alternative to [7, Theorem 14, p. 181]. Let $H(X)$ denote the convex hull of any set $X$ in $E^{3}$.

2.1. Suppose that $p \in E^{3}$ and that $X_{1}, \cdots, X_{n}$ are compact subsets of $E^{3}$ satisfying

(1) If $i, j$, and $k$ are distinct indices, then $X_{i} \cap X_{j} \cap X_{k}=\varnothing$.

(2) If $X_{i} \cap X_{j} \neq \varnothing$, then $H\left(X_{i} \cup X_{j}\right) \subset E^{3}-\{p\}$. Then $X=\bigcup_{i} X_{i}$ does not separate $p$ from infinity.

Proof. By [13, Theorem VI 10, p. 97], it suffices to show that $X$ is nullhomotopic in $E^{3}-\{p\}$. The sets $X_{1}, \cdots, X_{n}$ cover $X$; let $N$ be the nerve of that covering: one vertex $v_{i}$ for each nonempty $X_{i}$, one 1-simplex $v_{i} v_{j}$ for each nonempty intersection $X_{i} \cap X_{j}$. The nerve $N$ contains no higher dimensional simplexes by (1). The vertex $v_{i}$ can be realized geometrically as a point of $X_{i}$, the simplex $v_{i} v_{j}$ as the straight line segment in $H\left(X_{i} \cup X_{j}\right)$ joining $v_{i}$ and $v_{j}$. Map the sets $X_{i} \cap X_{j}$ to the midpoints of the 1-simplexes $v_{i} v_{j}$ and extend these partial maps on the sets $X_{i} \cap X_{j}$ to maps from the sets $X_{k}$ into the stars of the vertices $v_{k}$ in $N$ (the star is an absolute retract). This map can be realized as the final stage of a straight line homotopy in $E^{3}$ from $X$ into the (possibly singular) geometric realization of $N$ in $\bigcup\left\{H\left(X_{i} \cup X_{j}\right) \mid X_{i} \cap X_{j} \neq \varnothing\right\}$. The homotopy moves $X$ only in $\bigcup\left\{H\left(X_{i} \cup X_{j}\right) \mid X_{i} \cap X_{j} \neq \varnothing\right\} \subset E^{3}-\{p\}$. Since the geometric realization of $N$ is nullhomotopic in $E^{3}-\{p\}$, the proof is complete.

2.2. Suppose $S$ is a 2-sphere in $E^{3}$ and $\varepsilon>0$. Then there is a $\delta>0$ such that if $K$ is a compact set in $S$ and $K$ has no component of diameter $>\delta$, there exists a finite number of disjoint $\varepsilon$-disks $E_{1}, \cdots, E_{n}$ in $S$ such that $K \subset \bigcup_{i}$ Int $E_{i}$.

Proof. This can be deduced easily either from [9, Theorem 4.8.7] or its proof.

2.3. Addendum to the S.A.T. [7, §7]. The following conclusion may be added to the S.A.T.: There are disjoint $\varepsilon$-disks $E_{1}, \cdots, E_{m}$ on $h(S)$ such that

(3) $h(S)-\bigcup_{j} E_{j} \subset \operatorname{Int} S$. 
Proof. Choose $p \in \operatorname{Int} S$. We may assume that $\rho(p, S)>2 \varepsilon$. By (2.2), there is a $\delta, 0<\delta<\varepsilon$, such that if $K$ is a compact subset in $S$ having no component of diameter greater than $3 \delta$, there exist disjoint $\varepsilon / 3$-disks $E_{1}, \cdots, E_{m}$ in $S$ such that $K \subset \bigcup_{i}$ Int $E_{i}$.

By the S.A.T., there are disjoint $\delta$-disks $D_{1}, \cdots, D_{n}$ in $S$ and a $\delta$-homeomorphism from $S$ into $E^{3}$ such that

(1) $h(S)$ is polyhedral, and

(2) $S-\bigcup_{i} D_{i} \subset \operatorname{Ext} h(S)$.

Let $K_{0}=h(S) \cap \bigcup_{i} D_{i}$ and $K=h^{-1}\left(K_{0}\right)$. Then $K$ has no component of diameter greater than $3 \delta$ and is covered by the interiors of disjoint $\varepsilon / 3$-disks $E_{1}^{\prime}, \cdots, E_{m}^{\prime}$ in $S$. Let $E_{j}=h\left(E_{j}^{\prime}\right)$. Then $E_{1}, \cdots, E_{m}$ are disjoint $\varepsilon$-disks on $h(S)$ such that $S \cap h(S) \subset \bigcup_{j}$ Int $E_{j}$. It remains only to show that $h(S)-\bigcup_{j} E_{j} \subset \operatorname{Int} S$. Since $\rho(p, S \cup h(S))>\varepsilon$ and $S$ is homeomorphically within $\varepsilon$ of $S, p \in \operatorname{Int} h(S)$ [13, Theorem VI 10, p. 97]. Since $\rho(\mathrm{p}, S \cup h(S))>\varepsilon$ and each $D_{i}$ and $E_{j}$ has diameter less than $\varepsilon$, it follows from (2.1) that $\left(\bigcup_{i} D_{i}\right) \cup\left(\bigcup_{j} E_{j}\right)$ does not separate $p$ from infinity. Let $R$ be a ray from $p$ to infinity in $E^{3}-$ $\left[\left(\bigcup_{i} D_{i}\right) \cup\left(\bigcup_{j} E_{j}\right)\right]$. Let $q$ be the first point of $R \cap(S \cup h(S))$. If one had $q \in S$, then one would have a contradiction to (2) $S-\bigcup_{i} D_{i} \subset$ Ext $h(S)$. Hence $q \in h(S)$ and (3) $h(S)-\mathbf{U}_{j} E_{j} \subset$ Int $S$. This completes the proof.

S.A.T. for open subsets of spheres $[7, \S 8],[8]$. If $U$ is an open subset of a 2-sphere in $E^{3}$ and $f$ is a positive continuous real function on $U$, then there exists a homeomorphism $h$ of $S$ into $E^{3}$, a locally finite collection of disjoint disks $\left\{D_{i}\right\}$ in $U$ and a locally finite collection of disjoint disks $\left\{E_{j}\right\}$ in $h(U)$ such that:

(1) $h$ is the identity on $S-U$.

(2) $\rho(x, h(x))<f(x)$ for each $x \in U$.

(3) $h(U)$ is locally polyhedral.

(4) $U-U$ Int $D_{i} \subset \operatorname{Ext} h(S)$.

(5) $h(U)-U$ Int $E_{i} \subset$ Int $S$.

(6) Diam $D_{i}<\min$ value of $f$ on $D_{i}$.

(7) Diam $E_{i}<\min$ value of $f$ on $h^{-1}\left(E_{i}\right)$.

Proof. The proof proceeds almost without change from that of the S.A.T. One chooses an infinite triangulation of $U$ rather than a finite triangulation of $S$. Otherwise the argument is entirely local and the desired result follows.

3. Proof of the H.A.T. (statement appears in $\S 1$.) Throughout this section we assume that $S$ is a 2 -sphere in $E^{3}$ which satisfies the hypothesis of the H.A.T.; i.e., for each $\varepsilon>0$, there is an $\varepsilon$-homeomorphism from $S$ into Int $S$. By the S.A.T., we may assume that the 
image of $S$ is a polyhedral 2-sphere in Int $S$ which we call a polyhedral e-approximation to $S$ in $\operatorname{Int} S$.

Definition. Suppose $K$ is a (curvilinear) simplicial complex in $S$. We say that $K$ can be polyhedrally collared from Int $S$ if there is an embedding $h: K \times[0,1] \rightarrow S \cup \operatorname{Int} S$ such that

(1) $h(k, 0)=k$ if $k \in K$.

(2) $h(K \times(0,1]) \subset \operatorname{Int} S$.

(3) $h \mid K \times(0,1]$ is locally piecewise linear. Then $h$ is called a polyhedral collar on $K$ from Int $S$. (We identify $K \times[0,1]$ with some abstract simplicial complex for the purpose of defining piecewise linear.)

3.1. Each simple closed curve $J$ in $S$ can be polyhedrally collared from Int $S$.

Proof. In outline, the S.A.T. for open subsets of spheres $(\S 2)$ implies that we can raise a blister near one of the disks on $S$ which is bounded by $J$; we can then use the polyhedral approximations to $S$ in Int $S$ supplied by the hypothesis of the H.A.T. to cut off the feelers of the blister which reach back through $S$ (see Figure 4).

In more detail, let $D$ be a disk in $S$ bounded by $J$. Let $f(x)=$ $\rho(x, S-D)$ for each $x \in \operatorname{Int} D$. By the S.A.T. for open subsets of spheres (§2), there exists a homeomorphism of $S$ into $E^{3}$, a locally finite collection of disjoint disks $\left\{D_{i}\right\}$ in Int $D$, and a locally finite collection of disjoint disks $\left\{E_{j}\right\}$ in $h(\operatorname{Int} D)$ such that:

(1) $h \mid S-$ Int $D=$ identity.

(2) $\rho(x, h(x))<f(x)$ for each $x \in \operatorname{Int} D$.

(3) $h(\operatorname{Int} D)$ is locally polyhedral.

(4) Int $D-\mathrm{U}$ Int $D_{i} \subset \operatorname{Ext} h(S)$.

(5) $h(\operatorname{Int} D)-\bigcup \operatorname{Int} E_{i} \subset \operatorname{Int} S$.

(6) Diam $D_{i}<\min$ value of $f$ on $D_{i}$.

(7) Diam $E_{i}<\min$ value of $f$ on $h^{-1}\left(E_{i}\right)$.

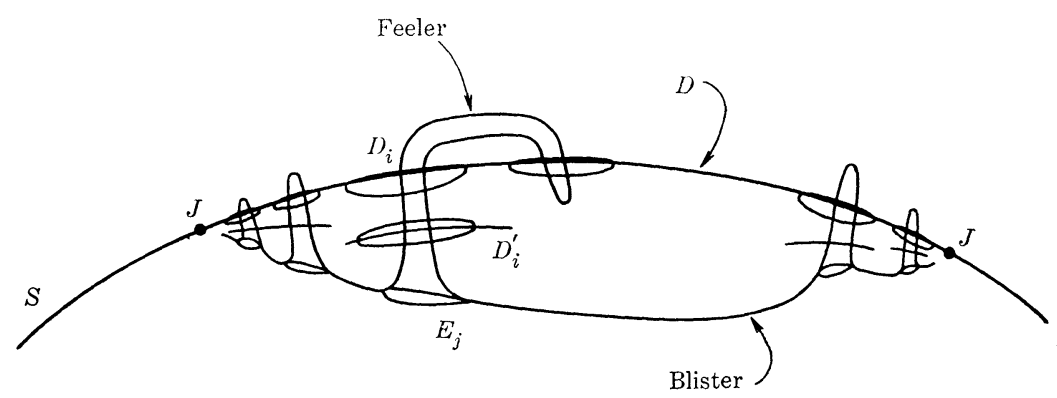

Int $S$

FIGURE 4 
Let $V$ denote the component of $h(\operatorname{Int} D)-S$ which has limit points in $J$. Note that $h(\operatorname{Int} D)-\mathrm{U}$ Int $E_{j} \subset V$. By the hypothesis of the H.A.T., $S$, and hence also each disk $D_{i}$, can be approximated very closely by a polyhedral set in Int $S$. It follows easily that there is a null sequence $D_{1}^{\prime}, D_{2}^{\prime}, \ldots$ of disjoint polyhedral disks in Int $S$, each $D_{i}^{\prime}$ approximating the corresponding disk $D_{i}$ and each in general position with respect to $h(S)$ such that

(8) $D_{i}^{\prime} \cap h(S) \subset \bigcup \operatorname{Int} E_{j}$.

(9) No component of $V-\cup D_{i}^{\prime}$ has limit points both in $J$ and in Int $D$.

Let $W$ be the component of $V-\cup D_{i}^{\prime}$ which has limit points in $J$. Note that $h(\operatorname{Int} D)-U \operatorname{Int} E_{j} \subset W$. Consider the set $\mathrm{Cl}(W)-W$. The components of this set form a null sequence $J, J_{1}, J_{2}, \cdots$ of simple closed curves, each $J_{i}$ lying in some Int $D_{j}^{\prime}$ and only finitely many of the $J_{i}$ in any one $D_{j}^{\prime}$. For those curves $J_{i}$ which lie in Int $D_{1}^{\prime}$, we fill in the holes in $\mathrm{Cl}(W)$ bounded by the $J_{i}$ with disks $D\left(J_{i}\right)$ in $D_{1}^{\prime}$ and push these disks slightly to one side of $D_{1}^{\prime}$, pushing those furthest which are innermost on $D_{1}^{\prime}$. If this is continued sequentially (say, in the order $\left.D_{1}^{\prime}, D_{2}^{\prime}, \cdots\right)$ and if care is exercised in the adjustments which push the disks $D\left(J_{i}\right)$ away from the disks $D_{j}^{\prime}$, one will obtain thereby a disk $E=\mathrm{Cl}(W) \cup D\left(J_{i}\right)$ (adjusted) which has $J$ as boundary and has interior which is a locally polyhedral subset of Int $S$. A polyhedral subset of Int $S$. A polyhedral collar for $J$ from Int $S$ can easily be identified in $E$.

3.2. If $K$ is a $\theta$-curve in $S$ (i.e., the union of three arcs with common endpoints and disjoint interiors) and $J$ is a simple closed curve in $K$, then any polyhedral collar on $J$ from Int $S$ can be extended to a polyhedral collar on $K$ from Int $S$.

Proof. Let $D$ be the disk in $S$ which is bounded by $J$ and contains the arc $\alpha=\mathrm{Cl}(K-J)$. Let $\beta$ be an arc which has the same endpoints $p$ and $q$ that $\alpha$ has and which lies except for $p$ and $q$ in $S-D$. Then $J^{\prime}=\alpha \cup \beta$ is a simple closed curve in $S$. Let $D^{\prime}$ be a disk bounded by $J^{\prime}$ in $S$.

We are given by hypothesis an annulus $A$ which polyhedrally collars $J$ from Int $S$. The annulus $A$ comes equipped with an assigned product structure $A=J \times[0,1](J=J \times\{0\})$ which we shall consider in more detail at the end of the proof. The proof of (3.1) showed how to construct a disk $E$ very near the disk $D^{\prime}$ such that $J^{\prime}=$ $\operatorname{Bd} E$, Int $E \subset$ Int $S$, and Int $E$ is locally polyhedral. It is clear from the proof of (3.1) (choose $f$ appropriately) that we may further require that $E \cap(J \times\{1\})=\varnothing$. We require that Int $E$ and $A$ be in general position. 
The inexperienced reader should compare the following discussion with [12]: Since Int $E$ and $A$ are in general position, there are only countably many components $K_{1}, K_{2}, \cdots$ of $A \cap$ Int $E$ and they form a null sequence, locally finite except possibly at $p$ and $q$ (the endpoints of $\alpha$ ), and satisfying for each individual $i$ either

(1) $\mathrm{Cl}\left(K_{i}\right)=K_{i}$ is a simple closed curve in Int $E$,

(2) $\mathrm{Cl}\left(K_{i}\right)=K_{i} \cup\{p\}$ or $K_{i} \cup\{q\}$ is a simple closed curve containing $p$ or $q$, or

(3) $\mathrm{Cl}\left(K_{i}\right)=K_{i} \cup\{p, q\}$ is an arc from $p$ to $q$.

If $E$ is chosen near enough to $D^{\prime}$, then is follows that each component $K_{i}$ of type (1) or (2) bounds a (unique) disk $A\left(K_{i}\right)$ in $A$. Such a $K_{i}$ also bounds a (unique) disk $E\left(K_{i}\right)$ in $E$. Let

$E_{0}=E-\bigcup\left\{\operatorname{Int} E\left(K_{i}\right) \mid K_{i}\right.$ is of type (1) or (2)\}, and
$E^{\prime}=E_{0} \cup\left[\bigcup\left\{\operatorname{Int} A\left(K_{i}\right) \mid K_{i}\right.\right.$ is of type (1) or (2) and $\left.\left.K_{i} \subset E_{0}\right\}\right] . \quad$ If $K_{i} \cup K_{j} \subset E_{0}(i \neq j), K_{i}$ and $K_{j}$ are of type (1) or (2), and $A\left(K_{i}\right) \subset A\left(K_{j}\right)$, then one is to understand that $E^{\prime}$ contains two copies of $A\left(K_{i}\right)-\{p, q\}$, one corresponding to $K_{i}$, the other lying in $A\left(K_{j}\right)$ and corresponding to $K_{j}$. With this understanding, $E^{\prime}$ is a singular disk which may be made nonsingular by pushing the sets $A\left(K_{i}\right)-\{p, q\}$ slightly to one side of $A$ in Int $S$. If $A\left(K_{i}\right) \subset A\left(K_{j}\right)$, then the copy of $A\left(K_{i}\right)$ corresponding to $K_{i}$ must be pushed further away from $A$ than is the copy of $A\left(K_{i}\right)$ in $A\left(K_{j}\right)$.

If each of the sets $A\left(K_{i}\right)-\{p, q\}$ is pushed to the appropriate side of $A$ and other appropriate care is taken, the nonsingular $E^{\prime}$ obtained will satisfy all of the conditions that the original $E$ satisfied except that there will be only finitely many components of $A \cap \operatorname{Int} E^{\prime}$ and each will be of type (3).

Since $J$ separates Int $\alpha=\alpha-\{p, q\}$ from Int $\beta$ in $S$, it follows that $A \cap \operatorname{Int} E^{\prime} \neq \varnothing$, hence that there is a disk $E^{\prime \prime}$ in $E^{\prime}$ such that $E^{\prime \prime} \cap A$ is a single arc in $A \cap \mathrm{Bd} E^{\prime \prime}$ from $p$ to $q$ and such that the complementary are in $\mathrm{Bd} E^{\prime \prime}$ is the original are $\alpha$. Standard cut and paste techniques allow one to adjust $E^{\prime \prime}$ so that the arc $A \cap \mathrm{Bd} E^{\prime \prime}$ runs up the fiber $\{p\} \times[0,1]$ in $A=J \times[0,1]$, arond the upper rim $J \times\{1\}$ of $A$, and back down the fiber $\{q\} \times[0,1]$. The desired polyhedral collar for $K$ from Int $S$ can be identified in $A \cup E^{\prime \prime}$ (adjusted). This completes the proof of (3.2).

3.3. CoRollary to (3.2). If $T$ is a curvilinear triangulation of $S$, then the 1-skeleton of $T$ can be polyhedrally collared from Int $S$. If $T^{\prime}$ is a curvilinear derived subdivision of $T$, then any polyhedral collar from Int $S$ on the 1-skeleton of $T$ can be extended to a polyhedral collar from Int $S$ on the 1-skeleton of $T^{\prime}$.

Proof. The 1-skeleton of a triangulation can be built up from an 
initial simple closed curve by adding arcs spanning the 2-cells of increasingly fine cellular subdivisions of $S$. Thus the corollary follows directly from (3.1) and (3.2).

3.4. Suppose $T$ is a curvilinear triangulation of $S, h:\left|T^{1}\right| \times[0,1] \rightarrow$ $S \cup \operatorname{Int} S$ is a polyhedral collar on the 1-skeleton $T^{1}$ of $T$ from Int $S$, and $\alpha$ and $\beta$ are positive numbers less than 1 . Then there is a polyhedral embedding $f: S \rightarrow \operatorname{Int} S$ such that:

(1) $f(S)$ separates $h\left(\left|T^{1}\right| \times\{1\}\right)$ from $S$ in $E^{3}$,

(2) $f(t)=h(t, \alpha)$ for each $t \in\left|T^{1}\right|$, and

(3) $f(\operatorname{Int} \sigma) \subset N(\sigma \cup h(\operatorname{Bd} \sigma \times[0, \alpha]) ; \beta)-h\left(\left|T^{1}\right| \times[0,1]\right)$ for each 2-simplex $\sigma \in T^{2}$.

Proof. Suppose that, for each 2-simplex $\sigma \in T^{2}$, we have shown how to construct a polyhedral disk $\sigma^{\prime}$ such that $\mathrm{Bd} \sigma=h(\mathrm{Bd} \sigma \times\{\alpha\})$ and Int $\sigma^{\prime} \subset N(\sigma \cup h(\mathrm{Bd} \sigma \times[0, \alpha]) ; \beta)-h\left(\left|T^{1}\right| \times[0,1]\right)$. Then, for $\beta$ sufficiently small (which we may certainly assume), $S^{\prime}=\bigcup\left\{\sigma^{\prime} \mid \sigma \in T^{2}\right\}$ will automatically be a polyhedral 2-sphere in $E^{3}$ which separates $h\left(\left|T^{1}\right| \times\{1\}\right)$ from $S$ in $E^{3}$; and a homeomorphism $f: S \rightarrow S^{\prime}$ satisfying the requirements of (3.4) can be constructed by defining $f \| T^{1} \mid$ by requirement (2) and then, for each $\sigma \in T^{2}$, extending $f \mid \mathrm{Bd} \sigma$ across Int $\sigma$ to take $\sigma$ to $\sigma^{\prime}$. Thus it suffices to construct $\sigma^{\prime}$.

Suppose that $\sigma$ is a 2-simplex in the 2-skeleton $T^{2}$ of $T$. Let $S_{0}$ be a polyhedral $\beta$-approximation to $S$ from Int $S$ with $h(\mathrm{Bd} \sigma \times[0,1])$ and $S_{0}$ in general position. For $\beta$ sufficiently small it will be true that

(4) $S_{0} \cap h(\mathrm{Bd} \sigma \times[0,1]) \subset h(\mathrm{Bd} \sigma \times(0, \alpha))$,

(5) $S_{0}$ separates $h(\mathrm{Bd} \sigma \times\{1\})$ from $S$, and

(6) some component $C$ of $S-h\left(\left|T^{1}\right| \times[0,1]\right)$ will lie in $N(\sigma ; \beta)$, have its boundary components in $N(\mathrm{Bd} \sigma \times[0,1])$, and have precisely one boundary component which is not contractible in the annulus $h(\mathrm{Bd} \sigma \times[0,1])$.

Fill in those boundary components of $C$ which are contractible in $h(\mathrm{Bd} \sigma \times[0,1])$ with the disks they bound in $h(\mathrm{Bd} \sigma \times[0, \alpha])$ and push them to the " $\sigma$ "-side of $h(\operatorname{Bd} \sigma \times[0, \alpha])$. This yields a disk $\sigma^{\prime}$ with $\mathrm{Bd} \sigma^{\prime}=K$ and Int $\sigma^{\prime} \subset \operatorname{Int} S-h\left(\left|T^{1}\right| \times[0,1]\right)$. Since the simple closed curves $K$ and $h(\mathrm{Bd} \sigma \times\{\alpha\})$ are parallel in $h(\mathrm{Bd} \sigma \times(0, \alpha])$, cut and paste can be used to slide $K$ upward in the annulus $h(\mathrm{Bd} \sigma \times[0, \alpha])$ so as to coincide with $h(\mathrm{Bd} \sigma \times\{\alpha\})$ as required. This completes the construction of $\sigma^{\prime}$ and the proof of (3.4).

Proof of the H.A.T. We now construct a homeomorphism from $S \cup \operatorname{Int} S$ to the unit ball $B^{3}$ in $E^{3}$ centered at the origin.

Let $T_{1}$ denote a curvilinear triangulation of $S$ and $T_{2}, T_{3}, \cdots$ 
successive curvilinear derived subdivisions of $T_{1}$ so chosen that mesh $T_{i} \rightarrow 0$ as $i \rightarrow \infty$.

By (3.3), there exist for $i=1,2, \cdots$ polyhedral collars $h_{i}:\left|\left(T_{i}\right)^{1}\right| \times$ $[0,1] \rightarrow S \cup \operatorname{Int} S$ on the 1-skeletons $\left(T_{i}\right)^{1}$ of these triangulations, $h_{i+1}$ extending $h_{i}$. We may clearly require that, for each $t \in\left|\left(T_{i}\right)^{1}\right|$, Diam $h_{i}\left(\left\{t_{i}\right\} \times[0,1 / i]\right)<1 / i$.

By (3.4), there exist for $i=1,2, \cdots$ polyhedral embeddings $f_{i}: S \rightarrow$ Int $S$ such that:

(1) $f_{i}(S)$ separates $h_{i}\left(\left|\left(T_{i}\right)^{1}\right| \times\{1\}\right)$ from $S$ in $E^{3}$,

(2) $f_{i}(t)=h_{i}(t, 1 / 2 i)$ for each $t \in\left|\left(T_{i}\right)^{1}\right|$, and

(3) $f_{i}(\operatorname{Int} \sigma) \subset N(\sigma, 1 / 2 i)-h_{i}\left(\left|\left(T_{i}\right)^{1}\right| \times[0,1]\right)$ for each 2-simplex $\sigma \in\left(T_{i}\right)^{2}$.

Let $n_{1}=1, n_{2}, \cdots$ be a subsequence of $1,2, \cdots$ so chosen that $f_{n_{i}}(S) \cap f_{n_{j}}(S)=\varnothing$ for $i \neq j$. Let $H_{n_{i}}$ be the restriction of the polyhedral collar $h_{n_{i}}$ to the set $\left|\left(T_{n_{i}}\right)^{1}\right| \times\left[0,1 / 2 n_{i}\right]$. Then

$$
X=S \cup\left\{\bigcup_{i} f_{n_{i}}(S)\right\} \cup\left\{\bigcup_{i} \text { Image } H_{n_{i}}\right\}
$$

is the template which we shall use to describe a homeomorphism

$$
h: S \cup \operatorname{Int} S \longrightarrow B^{3} \text {. }
$$

Description of $h \mid S$. Let $h \mid S: S \rightarrow\left(S^{2}=\mathrm{Bd} B^{3}\right)$ be any homeomorphism.

Description of $h \mid$ Image $H_{n_{i}}$. If $t \in\left|\left(T_{n_{i}}\right)^{1}\right|$ and $\alpha \in\left[0,1 / 2 n_{i}\right]$, let $h\left(H_{n_{i}}(t, \alpha)\right)$ be on the radius joining $h(t)$ and the origin in $B^{3}$ and at a distance $\alpha$ from $h(t)$.

Description of $h \mid f_{n_{i}}(S)$. If $s \in S$, let $h\left(f_{n_{i}}(s)\right)$ be on the radius joining $h(s)$ and the origin in $B^{3}$ and at a distance $1 / 2 n_{i}$ from $h(s)$.

Description of $h \mid(S \cup \operatorname{Int} S)-X$. Let $U$ be a component of $(S \cup \operatorname{Int} S)-X$. Then Bd $U$ is a polyhedral 2-sphere in $X$ of which $U$ is the interior. By Alexander's Theorem (see [2], [11] or [14]), $U \cup \mathrm{Bd} U$ is a 3-cell. The map $h \mid \mathrm{Bd} U$ is already defined and takes $\mathrm{Bd} U$ homeomorphically onto a curvilinear 2-sphere $h(\mathrm{Bd} U)$ in $\operatorname{Int} B^{3}$. Obviously, $h(\mathrm{Bd} U) \cup \operatorname{Int} h(\mathrm{Bd} U)$ is also a 3-cell. Since both $U \cup \operatorname{Bd} U$ and $h(\mathrm{Bd} U) \cup \operatorname{Int} h(\mathrm{Bd} U)$ are 3-cells, there is an extension of $h \mid \mathrm{Bd} U$ which takes $U \cup \operatorname{Bd} U$ homeomorphically onto $h(\operatorname{Bd} U) \cup \operatorname{Int} h(\operatorname{Bd} U)$. We take $h \mid U \cup \mathrm{Bd} U$ to be this homeomorphism.

One checks easily that $h$ defined piece by piece above defines a homeomorphism from $S \cup \operatorname{Int} S$ onto $B^{3}$. This completes the proof of the H.A.T. 
APPENDIX. We give an example of the type of argument one might use in establishing such things as the claims in Steps 1 and 3 of the proof of the S.A.T., Property (9) in the proof of (3.1), and the statements involving "for $\beta$ sufficiently small" in the proof of (3.4). Such details are often omitted as essentially obvious in the mathematical literature (or hidden under the rug, depending on one's viewpoint).

EXAMPLE (cf. the second paragraph of the proof of (3.4)). Let $S$ be a 2-sphere in $E^{3}, D$ a disk in $S$; and suppose $J=\mathrm{Bd} D$ has a polyhedral collar $h: J \times[0,1] \rightarrow S \cup \operatorname{Int} S$ from Int $S$. For each $\varepsilon>0$, there is a $\delta>0$ such that if $S$ has a polyhedral $\delta$-approximation $f: S \rightarrow$ Int $S$ from Int $S$ with $f(S)$ and $h(J \times[0,1])$ in general position, then:

(1) $f(S)$ separates $h(J \times[0,1])$ from $S$ in $E^{3}$, and

(2) there is a component $C$ of $f(S)-h(J \times[0,1])$ such that $C \subset N(D, \varepsilon)$ and $\mathrm{Cl}(C)-C$ has precisely one component in $h(J \times[0,1])$ which does not bound a disk in $h(J \times[0,1])$.

(Note that if $f(S) \cap h(J \times\{1\}) \neq \varnothing$ and if $C^{\prime}$ is any component of $f(S)-h(J \times[0,1])$, then $\mathrm{Cl}\left(C^{\prime}\right)$ is a disk-with-holes with interior equal to $C^{\prime}$ and boundary curves in $h(J \times[0,1])$.)

Proof. We first require an auxiliary construction: let $\alpha$ and $\beta$ be arcs with common endpoints $p \in \operatorname{Int} D$ and $g \in S-D$ such that $\alpha-\{p, q\} \subset \operatorname{Int} S-h(J \times[0,1])$ and $\beta-\{p, q\} \subset \operatorname{Ext} S$. Let $D_{p} \subset \operatorname{Int} D$ and $D_{q} \subset S-D$ be disks such that $p \in \operatorname{Int} D_{p}$ and $q \in \operatorname{Int} D_{q}$.

We choose $\delta>0$ and smaller than the following numbers

$$
\begin{aligned}
& \delta_{1}=\rho[S, h(J \times\{1\})] \\
& \delta_{2}=\rho\left[D_{p} \cup D_{q}, h(J \times[0,1])\right] \\
& \delta_{3}=\min \{\rho(p, S-D), \rho(q, D)\} \\
& \delta_{4}=\min \{\varepsilon / 2, \rho[S-N(D, \varepsilon / 2), h(J \times[0,1])]\} \\
& \delta_{5}=\rho\left(S-\left(\operatorname{Int} D_{p} \cup \operatorname{Int} D_{q}\right), \alpha \cup \beta\right] .
\end{aligned}
$$

(a) $f(S)$ separates $h(J \times\{1\})$ from $S$ in $E^{3}$ : Indeed, $\delta<\delta_{1}$ implies that $S$ and $f(S)$ are homotopic in $E^{3}-h(J \times\{1\})$, hence that $h(J \times\{1\}) \subset \operatorname{Int} f(S)$ by [13, Theorem VI 10, p. 94]. Since $S \subset \operatorname{Ext} f(S)$, the result follows.

(b) $f\left(D_{p}\right) \cap h(J \times[0,1])=\varnothing=f\left(D_{q}\right) \cap h(J \times[0,1])$ since $\delta<\delta_{2}$.

(c) The component $C$ of $f(S)-h(J \times[0,1])$ which contains $f\left(D_{p}\right)$ (such exists by (b)) does not contain $f\left(D_{q}\right)$ : Indeed, suppose $\{f(p), f(q)\} \subset C$. Let $\gamma$ be an arc in $C$ from $f(p)$ to $f(q)$. Let $\gamma_{p}$ and $\gamma_{q}$ be the straight line segments in $E^{3}$ joining $p$ to $f(p)$ and $f(q)$ to $q$, respectively. Let $\gamma^{\prime}$ be an arc from $q$ to $p$ in $\operatorname{Ext}(S) \cup\{p, q\}$. Because $\delta<\delta_{3}, \gamma_{p} \cap$ $S \subset$ Int $D$ and $\gamma_{q} \cap S \subset S-D$. It follows that the loop $\Gamma$ which 
traverses $\gamma, \gamma_{q}, \gamma^{\prime}$, and $\gamma_{p}$ (in that order) has linking number \pm 1 with $J$. Since $\delta<\delta_{2}, \Gamma \cap h(J \times[0,1])=\varnothing$. Hence $L(\Gamma, h(J \times\{1\}))= \pm 1$ by (0.5) or (0.6), and $\Gamma$ is noncontractible in $E^{3}-h(J \times\{1\})$ by $(0.5)$. But $\Gamma$ is homotopic in $E^{3}-h(J \times\{1\})$ to the loop $\Gamma^{\prime}$ which traverses first $f^{-1}(\gamma)$ and then $\gamma^{\prime}$ since $\delta<\delta_{1}$, and $\Gamma^{\prime}$ is contractible in the simply connected set $S \cup$ Ext $S$, a contradiction.

(d) $C \subset N(D, \varepsilon)$. The proof is like that of (c) with the following adjustments. One uses $q^{\prime}$ such that $f\left(q^{\prime}\right) \in C-N(D, \varepsilon)$ instead of the original $q$ in the construction of $\Gamma$. One uses the fact that $\delta<\delta_{4}$ to prove that $\gamma_{q}^{\prime} \cap S \subset S-D$ and that $\Gamma \cap h(J \times[0,1])=\varnothing$.

(e) $\mathrm{Cl}(C)-C$ has a component which bounds no disk in $h(J \times$ $[0,1])$. Indeed, $L\left(\mathrm{Bd} D_{p}, \alpha \cup \beta\right)= \pm 1$ by construction (cf. (0.7)). Also, $\mathrm{Bd} D_{p}$ and $f\left(\operatorname{Bd} D_{p}\right)$ are homotopic in $E^{3}-(\alpha \cup \beta)$ since $\delta<\delta_{5}$. Thus $L\left(f\left(\operatorname{Bd} D_{p}\right), \alpha \cup \beta\right)= \pm 1$. If each component of $\mathrm{Cl}(C)-C$ bounded a disk in $h(J \times[0,1])$, then $f\left(\operatorname{Bd} D_{p}\right)$ would bound a singular disk in $\left[C-\right.$ Int $\left.D_{p}\right] \cup h(J \times[0,1]) \subset E^{3}-(\alpha \cup \beta)$, a contradiction to $(0.5)$.

(f) $\mathrm{Cl}(C)-C$ has at most one component which bounds no disk in $h(J \times[0,1])$. For otherwise, at least one such component would bound a disk in $f(S)-\left(D_{p} \cup D_{q}\right) \subset E^{3}-(\alpha \cup \beta)$ even though it would link $\alpha \cup \beta$, a contradiction.

Added in proof. O. G. Harrold has pointed out an unwarranted assumption at the beginning of [12] (cf. paragraph 3 of the proof of our Lemma 3.2) which invalidates the main result of [12] but does not invalidate the very nice treatment of infinite cut and paste techniques in [12]. Indeed, the false assumption of [12] is valid in many situations, including ours (i.e., the paragraph cited above is correct). L. Keldyš has also claimed the main result of [12], her proof is also incorrect [Math. USSR Sbornik, 10 (1970), 267-287]. The status of the main result of [12] is apparently undecided.

\section{REFERENCES}

1. P. S. Aleksandrov, Combinatorial Topology, Vol. 3, OGIZ, Moscow, 1947; English transl., Graylock Press, Albany, New York, 1960.

2. J. W. Alexander, On the subdivision of 3-space by a polyhedron, Proc. Nat. Acad. Sci., U.S.A., 10 (1924), 6-8.

3. Steve Armentrout, Cellular decompositions of 3-manifolds that yield 3-manifolds, Memoirs of the Amer. Math. Soc., No. 107, Amer. Math. Soc., Providence, Rhode Island, 1971.

4. R. H. Bing, Approximating surfaces with polyhedral ones, Ann. of Math., (2) 65 (1957), 456-483.

5. Conditions under which a surface in $E^{3}$ is tame, Fund. Math., 47 (1959), 105-139.

6. - An alternative proof that 3-manifolds can be triangulated, Ann. of Math., (2) 69 (1959), 37-65. 
7. R. H. Bing, Approximating surfaces from the side, Ann. of Math., (2) 77 (1963), 143-192.

8. - Improving the side approximation theorem, Trans. Amer. Math. Soc., 116 (1965), 511-525.

9. C. E. Burgess and J. W. Cannon, Embeddings of surfaces in $E^{3}$, Rocky Mountain J. Math., 1 (1971), 259-344.

10. J. W. Cannon, ULC properties in neighbourhoods of embedded surfaces and curves in $E^{3}$, Canad. J. Math., 25 (1973), 31-73.

11. W. Graeub, Die semilineare Abbildungen, S.-B. Heidelberger Akad. Wiss. Math.Nat. Kl., (1950), 205-272.

12. H. C. Griffith and O. G. Harrold, Simplifying intersections of surfaces, Proc. Nat. Acad. Sci., U.S.A., 65 (1970), 837-840.

13. W. Hurewicz and H. Wallman, Dimension Theory, Princeton Math. Series, vol. 4, Princeton Univ. Press, Princeton, N. J., 1941.

14. E. E. Moise, Affine structures in 3-manifolds. II. Positional Properties of 2-spheres, Ann. of Math., (2) 55 (1952), 172-176.

15. - Affine structures in 3-manifolds. $V$. The triangulation theorem and Hauptvermutung, Ann. of Math., (2) 56 (1952), 96-114.

16. C. D. Papakyriakopoulos, On Dehn's lemma and the asphericity of knots, Ann. of Math., (2) 66 (1957), 1-26.

17. - On solid tori, Proc. London Math. Soc., (3) 7 (1957), 281-299.

18. H. Seifert and W. Threlfall, Lehrbuch der Topologie, Leipzig, Teubner, 1934.

19. R. L. Wilder, Topology of manifolds, Amer. Math. Soc. Colloq. Publ., vol. 32, Amer. Math. Soc., Providence, Rhode Island, 1963.

Received February 7, 1972. Presented to the American Mathematical Society in Madison, Wisconsin, April, 1970. The author is a Sloan Foundation Research Fellow.

INSTITUTE FOR ADVANCED STUdY

AND

UNIVERSITY OF WISCONSIN AT MADISON 



\section{PACIFIC JOURNAL OF MATHEMATICS}

\section{EDITORS}

D. Gilbarg and J. Milgram

Stanford University

Stanford, California 94305

\author{
R. A. Beaumont \\ University of Washington \\ Seattle, Washington 98105
}

J. DuGUNDJI

Department of Mathematics

University of Southern California

Los Angeles, California 90007

Richard ARENS

University of California

Los Angeles, California 90024

\section{ASSOCIATE EDITORS}
E. F. BECKENBACH
B. H. NeumanN
F. WOLF
K. YosHIDA

\section{SUPPORTING INSTITUTIONS}

UNIVERSITY OF BRITISH COLUMBIA

CALIFORNIA INSTITUTE OF TECHNOLOGY

UNIVERSITY OF CALIFORNIA

MONTANA STATE UNIVERSITY

UNIVERSITY OF NEVADA

NEW MEXICO STATE UNIVERSITY

OREGON STATE UNIVERSITY

UNIVERSITY OF OREGON

OSAKA UNIVERSITY

\author{
UNIVERSITY OF SOUTHERN CALIFORNIA \\ STANFORD UNIVERSITY \\ UNIVERSITY OF TOKYO \\ UNIVERSITY OF UTAH \\ WASHINGTON STATE UNIVERSITY \\ UNIVERSITY OF WASHINGTON
* * * *
AMERICAN MATHEMATICAL SOCIETY \\ NAVAL WEAPONS CENTER
}

The Supporting Institutions listed above contribute to the cost of publication of this Journal, but they are not owners or publishers and have no responsibility for its content or policies.

Mathematical papers intended for publication in the Pacific Journal of Mathematics should be in typed form or offset-reproduced, (not dittoed), double spaced with large margins. Underline Greek letters in red, German in green, and script in blue. The first paragraph or two must be capable of being used separately as a synopsis of the entire paper. The editorial "we" must not be used in the synopsis, and items of the bibliography should not be cited there unless absolutely necessary, in which case they must be identified by author and Journal, rather than by item number. Manuscripts, in duplicate if possible, may be sent to any one of the four editors. Please classify according to the scheme of Math. Rev. Index to Vol. 39. All other communications to the editors should be addressed to the managing editor, Richard Arens, University of California, Los Angeles, California, 90024.

50 reprints are provided free for each article; additional copies may be obtained at cost in multiples of 50 .

The Pacific Journal of Mathematics is issued monthly as of January 1966. Regular subscription rate: $\$ 48.00$ a year (6 Vols., 12 issues). Special rate: $\$ 24.00$ a year to individual members of supporting institutions.

Subscriptions, orders for back numbers, and changes of address should be sent to Pacific Journal of Mathematics, 103 Highland Boulevard, Berkeley, California, 94708.

PUBLISHED BY PACIFIC JOURNAL OF MATHEMATICS, A NON-PROFIT CORPORATION

Printed at Kokusai Bunken Insatsusha (International Academic Printing Co., Ltd.), 270, 3-chome Totsuka-cho, Shinjuku-ku, Tokyo 160, Japan. 


\section{Pacific Journal of Mathematics}

\section{Vol. 46, No. 2 December, 1973}

Christopher Allday, Rational Whitehead products and a spectral sequence of

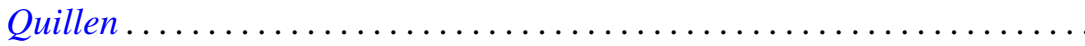

James Edward Arnold, Jr., Attaching Hurewicz fibrations with fiber

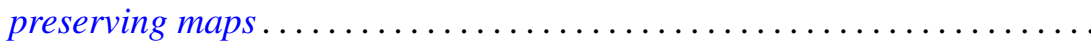

Catherine Bandle and Moshe Marcus, Radial averaging transformations with various metrics.................................

David Wilmot Barnette, A proof of the lower bound conjecture for convex

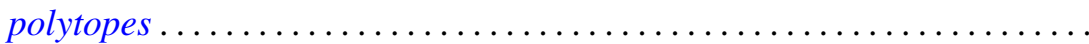

Louis Harvey Blake, Simple extensions of measures and the preservation of

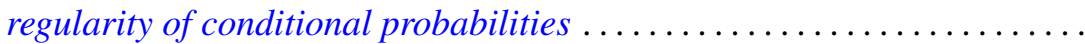

James W. Cannon, New proofs of Bing's approximation theorems for

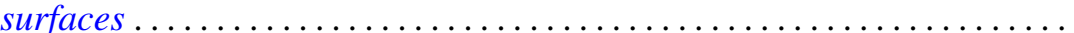

C. D. Feustel and Robert John Gregorac, On realizing HNN groups in

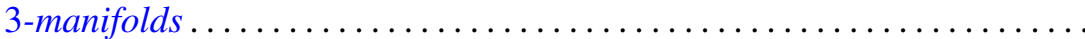

Theodore William Gamelin, Iversen's theorem and fiber algebras . . . . . . . . 389

Daniel H. Gottlieb, The total space of universal fibrations . . . . . . . . . . . .

Yoshimitsu Hasegawa, Integrability theorems for power series expansions of

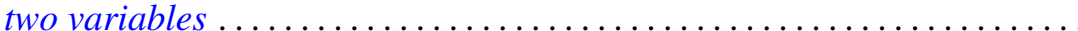

Dean Robert Hickerson, Length of period simple continued fraction expansion of $\sqrt{ } d$

Herbert Meyer Kamowitz, The spectra of endomorphisms of the disc algebra.

Dong S. Kim, Boundedly holomorphic convex domains

Daniel Ralph Lewis, Integral operators on $\mathscr{L}_{p}$-spaces ...

John Eldon Mack, Fields of topological spaces . . . . . . . . .

V. B. Moscatelli, On a problem of completion in bornology

Ellen Elizabeth Reed, Proximity convergence structures. .

Ronald C. Rosier, Dual spaces of certain vector sequence spaces .

Robert A. Rubin, Absolutely torsion-free rings

Leo Sario and Cecilia Wang, Radial quasiharmonic functions . .

James Henry Schmerl, Peano models with many generic classes .

H. J. Schmidt, The $\mathscr{F}$-depth of an $\mathscr{F}$-projector ............

Edward Silverman, Strong quasi-convexity. . . . . . . . . . . . . . . . . 549

Barry Simon, Uniform crossnorms ....................... 555

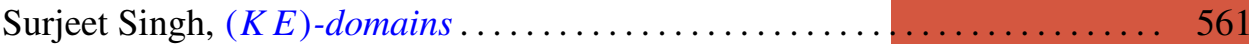

Ted Joe Suffridge, Starlike and convex maps in Banach spaces . . . . . . . . 575

Milton Don Ulmer, $C$-embedded $\Sigma$-spaces . . . . . . . . . . . . . . . . 591

Wolmer Vasconcelos, Conductor, projectivity and injectivity . . . . . . . . . 603 